\title{
SOME SHARP HODGE LAPLACIAN AND STEKLOV EIGENVALUE ESTIMATES FOR DIFFERENTIAL FORMS
}

\author{
KWOK-KUN KWONG
}

\begin{abstract}
We give some sharp lower bounds of the first eigenvalue for the Hodge Laplacian acting on differential forms on the boundary of a Riemannian manifold. We also give some sharp estimates for the first nonzero Steklov eigenvalue for differential forms.
\end{abstract}

\section{INTRODUCTION}

In this paper, we obtain some sharp lower bounds for the first nonzero Hodge Laplacian eigenvalue and also Steklov eigenvalue for differential forms on a boundary $\Sigma$ of a compact Riemannian manifold $(N, g)$ in terms of the extrinsic curvature of $\Sigma$ and the intrinsic curvature of $N$. The main tools we use are Hodge theory and a Reilly formula ([15]) for differential forms on a manifold with boundary. Our main results, Theorem 2.3 and 2.4, are generalizations of the results of ChoiWang [3], Escobar [4], Xia [24], Wang-Xia [22] and Raulot-Savo [15]. For instance, in Theorem 2.3 we generalize the results of Xia [24] and Raulot-Savo [15]:

Theorem 1.1. Let $\left(N^{n}, g\right)$ be a compact orientable Riemannian manifold with boundary $\Sigma$. Suppose the Bochner curvature $W^{r}$ or $W^{n-r}$ on $N$ is bounded from below by $k \geq 0$. Assume that the lowest q-curvature $s_{q}$ of $\Sigma$ is nonnegative, where $q=\min \{r, n-r\}$. Then for $1 \leq r \leq n-1$, we have

$$
2 \lambda_{1, r}^{\prime}=2 \lambda_{1, r-1}^{\prime \prime} \geq k+s_{r} s_{n-r}+\sqrt{\left(s_{r} s_{n-r}\right)^{2}+2 s_{r} s_{n-r} k},
$$

where $\lambda_{1, r}^{\prime}$ (resp. $\lambda_{1, r}^{\prime \prime}$ ) is the first nonzero eigenvalue of the Hodge Laplacian on the exact (resp. co-exact) $r$-forms on $\Sigma$. The equality can

2010 Mathematics Subject Classification. 53C20, 53C24, 53C40.

Key words and phrases. Hodge Laplacian, differential forms, Steklov eigenvalue, Reilly's formula.

Research partially supported by Ministry of Science and Technology in Taiwan under grant MOST103-2115-M-006-016-MY3. 
hold only when $k=0$, with the $r$-curvatures and the $(n-r)$-curvatures being positive constants. If, furthermore, $(N, g)$ has non-negative Ricci curvature, then the equality holds if and only if $(N, g)$ is isometric to a Euclidean ball.

The curvatures $W^{r}$ and $s_{r}$ will be explained in Section 2, When $r=1, s_{1}$ is the minimum eigenvalue of the second fundamental form of $\Sigma$ and $s_{n-1}$ is the minimum of its mean curvature, $W^{1}$ is just the Ricci curvature and $\lambda_{1}=\lambda_{1,0}^{\prime \prime}$ is the first nonzero eigenvalue of the Laplacian on functions on $\Sigma$.

We will also give a sharp lower bound of $\lambda_{1, r}^{\prime}$ in terms of the first nonzero Steklov eigenvalues for differential forms, as well as some lower and upper bounds for the Steklov eigenvalues in terms of $\lambda_{1, r}^{\prime}$ (Theorem 2.4). The Steklov eigenvalue is the eigenvalue of an elliptic nonnegative self-adjoint pseudo-differential operator of order one, which will be explained in Section 2. Recently, there are a number of authors studying the Steklov eigenvalues problems (e.g. [5], 7], [8, [9]). It is also interesting to see that when $n=2$, an extension of the result of HangWang [11] gives an improvement of Choi-Wang's result [3], which is a special case of Theorem 2.3 . Indeed, we can prove that $\lambda_{1}(\Sigma) \geq k$ and the estimate is sharp (Theorem 3.1). It may have some independent interest.

There are a number of applications of our results. For example:

Theorem 1.2. (Corollary 3.1) If $\Sigma$ is a closed surface of genus $g$ in $\mathbb{S}^{3}$ with second fundamental form bounded from below by $s_{1}$ and mean curvature bounded from below by $s_{n-1}$, then

$$
\left(2+s_{n-1} s_{1}+\sqrt{\left(s_{n-1} s_{1}\right)^{2}+4 s_{n-1} s_{1}}\right) \text { Area }(\Sigma)<16 \pi(g+1) .
$$

This paper is organized as follows. In Section 2, we prove the various estimates for the Hodge Laplacian eigenvalues and also Steklov eigenvalues for differential forms on a manifold with boundary. In Section 3. we give some applications of the main results and take a closer look when $N$ is 2-dimensional.

Acknowledgments: We would like to thank Prof. Luen-Fai Tam and Gilbert Weinstein for useful comments. We would also like to thank the anonymous referee for the useful comments and suggestions.

\section{Eigenvalue estimates}

In this section, we will prove several lower bounds of the first nonzero eigenvalue of the Hodge Laplacian on differential forms on the boundary 
$\Sigma$ of a Riemannian manifold $(N, g)$. These results are the natural generalizations of some results in [4], [11], [13], [15], [22] and [24].

Let us first set up the notations. Throughout this paper, $(N, g)$ denotes a compact $n$-dimensional connected oriented Riemannian manifold $(n \geq 2)$ smooth boundary $\partial N=\Sigma$. We denote the Levi-Civita connection on $N$ and $\Sigma$ by $\bar{\nabla}$ and $\nabla$ respectively.

Fix $x \in \Sigma$ and let $k_{1}(x), \cdots, k_{n-1}(x)$ be the principal curvatures of $\Sigma$ at $x$ w.r.t. the outward unit normal $\nu$. We define the $r$-curvatures (not to be confused with the $r$-th mean curvature) to be all the possible sums $k_{i_{1}}(x)+\cdots+k_{i_{r}}(x)$ where $i_{1}<\cdots<i_{r}$. We can assume $k_{1}(x) \leq$ $\cdots \leq k_{n-1}(x)$, then we define the lowest $r$-curvature to be

$$
s_{r}(x)=k_{1}(x)+\cdots+k_{r}(x) .
$$

We also define

$$
s_{r}(\Sigma)=\min _{x \in \Sigma} s_{r}(x) .
$$

Note that the second fundamental form is bounded from below by $s_{1}$ and $s_{n-1}(x)=H$ is the mean curvature. It is easy to see that if $l \leq m$, then $\frac{s_{l}}{l} \leq \frac{s_{m}}{m}$ and that $s_{l} \geq 0$ implies $s_{m} \geq 0$.

We denote by $\bar{d}$ and $\bar{\delta}$ the exterior derivative and its (formal) adjoint w.r.t. the $L^{2}$ inner product on $(N, g)$ respectively. The Hodge Laplacian $\bar{\Delta}$ of a $p$-form on $(N, g)$ is defined by

$$
\bar{\Delta} \alpha=-(\bar{d} \bar{\delta}+\bar{\delta} \bar{d}) \alpha
$$

for $\alpha \in \Omega^{r}(N)$. Our sign is chosen such that $\bar{\Delta}$ is the second derivative for functions on $N=\mathbb{R}$. Recall the Bochner formula (see e.g. [14] p.218 Theorem 50):

$$
-\bar{\Delta} \alpha=\bar{\nabla}^{*} \bar{\nabla} \alpha+W^{r}(\alpha)
$$

where $W^{r}$ is a self-adjoint endomorphism on $\Omega^{r}(N)$, which is determined by the Riemann curvature tensor on $(N, g)$. This term is called the Bochner curvature. When $r=1, W^{1}$ is just the Ricci curvature $\overline{\text { Ric }}$ of $N$ and by [10], $W^{r} \geq r(n-r) \gamma$ where $\gamma$ is the lowest eigenvalue of the curvature operator on $(N, g)$. However, $W^{r} \geq 0$ is usually much weaker than the curvature operator being nonnegative.

We define the shape operator $S=\bar{\nabla} \nu$ on $T \Sigma$ and define $S^{r}: \Omega^{r}(\Sigma) \rightarrow$ $\Omega^{r}(\Sigma)$ by

$$
S^{r} \alpha\left(X_{1}, \cdots, X_{r}\right)=\sum_{j=1}^{r} \alpha\left(X_{1}, \cdots, S\left(X_{j}\right), \cdots, X_{r}\right) .
$$


We also define $S^{0}$ to be zero. For example, if $\alpha$ is a 1 -form, then $S^{1} \alpha(X)=\alpha(S(X))$. Observe that $S^{n-1} \alpha=H \alpha$ and that the eigenvalues of $S^{r}$ are exactly the $r$-curvatures of $\Sigma$, therefore

$$
\left\langle S^{r} \alpha, \alpha\right\rangle \geq s_{r}(\Sigma)|\alpha|^{2} .
$$

We define $\lambda_{k, r}^{\prime}$ (respectively $\lambda_{k, r}^{\prime \prime}$ ) to be the $k-t h$ nonzero eigenvalue for the exact (respectively co-exact) $r$-forms on $\Sigma$. By Hodge decomposition theorem and Hodge duality (e.g. [23]), we have

$$
\left\{\begin{array}{l}
\lambda_{1, r}(\Sigma)=\min \left\{\lambda_{1, r}^{\prime}(\Sigma), \lambda_{1, r}^{\prime \prime}(\Sigma)\right\} \\
\lambda_{1, r}^{\prime \prime}(\Sigma)=\lambda_{1, r+1}^{\prime}(\Sigma) \\
\lambda_{1, r}^{\prime \prime}(\Sigma)=\lambda_{1, n-1-r}^{\prime}(\Sigma) .
\end{array}\right.
$$

From this we see that to determine $\lambda_{1, r}$, it suffices to determine $\lambda_{1, r}^{\prime}$ for $1 \leq r \leq\left\lfloor\frac{n}{2}\right\rfloor$.

The following formula is the generalization of Reilly's formula to differential forms.

Theorem 2.1. (15] Theorem 3) Let $\alpha \in \Omega^{r}(N), r \geq 1$, then

$$
\int_{N}\left(|\bar{d} \alpha|^{2}+|\bar{\delta} \alpha|^{2}-|\bar{\nabla} \alpha|^{2}\right)=\int_{N} W^{r}(\alpha, \alpha)-2 \int_{\Sigma}\left\langle\iota_{\nu} \alpha, \delta i^{*} \alpha\right\rangle+\int_{\Sigma} B(\alpha, \alpha)
$$

where the boundary term is given by

$$
B(\alpha, \alpha)=\left\langle S^{r}\left(i^{*} \alpha\right), i^{*} \alpha\right\rangle+\left\langle S^{n-r}\left(i^{*} \bar{*} \alpha\right), i^{*} \bar{*} \alpha\right\rangle .
$$

Here $i: \Sigma \rightarrow N$ is the inclusion and $₹: \Omega^{r}(N) \rightarrow \Omega^{n-r}(N)$ is the Hodge star operator on $N$. We will also denote by $d$ and $\delta$ the exterior derivative and its adjoint on $\Sigma$ respectively.

The classical Reilly formula can be recovered by setting $\alpha=\bar{d} f \in$ $\Omega^{1}(N)$ :

Theorem 2.2. 18] Let $f$ be a smooth function on $N$ and $z=\left.f\right|_{\Sigma}$. Then

$$
\int_{N}\left((\bar{\Delta} f)^{2}-\left|\bar{\nabla}^{2} f\right|^{2}\right)=\int_{N} \overline{\operatorname{Ric}}(\bar{\nabla} f, \bar{\nabla} f)+\int_{\Sigma}\left(2 \frac{\partial f}{\partial \nu} \Delta z+H\left(\frac{\partial f}{\partial \nu}\right)^{2}+A(\nabla z, \nabla z)\right) .
$$

Here $A$ is the second fundamental form and $H=\operatorname{tr}_{\Sigma}(A)$ is the mean curvature of $\Sigma$ in $N$.

We now state our first main result. 
Theorem 2.3. Let $\left(N^{n}, g\right)$ be a compact orientable Riemannian manifold with boundary $\Sigma$. Suppose $W^{r}$ or $W^{n-r}$ on $N$ is bounded from below by $k \geq 0$. Assume that $s_{q} \geq 0$ where $q=\min \{r, n-r\}$. Then for $1 \leq r \leq n-1$, we have

$$
2 \lambda_{1, r}^{\prime}=2 \lambda_{1, r-1}^{\prime \prime} \geq k+s_{r} s_{n-r}+\sqrt{\left(s_{r} s_{n-r}\right)^{2}+2 s_{r} s_{n-r} k} .
$$

If the equality holds, then $k=0$, the $r$-curvatures constantly equal $s_{r}>$ 0 and the $(n-r)$-curvatures constantly equal $s_{n-r}>0$. If, furthermore, $(N, g)$ has non-negative Ricci curvature, then the equality holds if and only if $(N, g)$ is isometric to a Euclidean ball. The condition on Ricci curvature can be removed if $r=1$ or $n-1$.

Proof. Note that by Hodge decomposition theorem and Hodge duality, $\lambda_{1, r}^{\prime}=\lambda_{1, r-1}^{\prime \prime}=\lambda_{1, n-r}^{\prime}$ and by (2.6) below, both $W^{r}$ and $W^{n-r}$ are bounded from below by $k$.

Let $\phi$ be a co-exact $(r-1)$ eigenform on $\Sigma$ with eigenvalue $\lambda=$ $\lambda_{1, r-1}^{\prime \prime}=\lambda_{1, r}^{\prime}$, i.e. $\Delta \phi=-\delta d \phi=-\lambda \phi$. Then $\omega=d \phi$ is an exact $r$ eigenform with eigenvalue $\lambda$. By Theorem 2 of [2] (p.148), there exists an $(r-1)$-form $\bar{\phi}$ on $N$ such that $\bar{\delta} \bar{d} \bar{\phi}=0$ and $i^{*} \bar{\phi}=\phi$ on $\Sigma$. Let $\bar{\omega}=\bar{d} \bar{\phi}$. Then

$$
\left\{\begin{array}{l}
\bar{d} \bar{\omega}=\bar{\delta} \bar{\omega}=0 \quad \text { on } N \\
i^{*} \bar{\omega}=\omega \quad \text { on } \Sigma .
\end{array}\right.
$$

Using Reilly's formula on $\bar{\omega}=\bar{d} \bar{\phi}$,

$$
\begin{aligned}
0 & \geq \int_{N}-|\bar{\nabla} \bar{\omega}|^{2} \\
& =\int_{N} W^{r}(\bar{d} \bar{\phi}, \bar{d} \bar{\phi})+\int_{\Sigma}-2\left\langle\iota_{\nu} \bar{\omega}, \delta \omega\right\rangle+\left\langle S^{r}\left(i^{*} \bar{\omega}\right), i^{*} \bar{\omega}\right\rangle+\left\langle S^{n-r}\left(i^{*} \bar{\kappa} \bar{\omega}\right), i^{*} \bar{\omega} \bar{\omega}\right\rangle \\
& \geq k \int_{N}|\bar{d} \bar{\phi}|^{2}+\int_{\Sigma}-2 \lambda\left\langle\iota_{\nu} \bar{\omega}, \phi\right\rangle+s_{r}\left|i^{*} \bar{\omega}\right|^{2}+s_{n-r}\left|i^{*} \bar{\omega}\right|^{2} \\
& =k \int_{N}\langle\bar{\phi}, \bar{\delta} \bar{d} \bar{\phi}\rangle+k \int_{\Sigma}\left\langle i^{*} \bar{\phi}, \iota_{\nu} \bar{d} \bar{\phi}\right\rangle+\int_{\Sigma}-2 \lambda\left\langle\iota_{\nu} \bar{\omega}, \phi\right\rangle+s_{r}|\omega|^{2}+s_{n-r}\left|i^{*} \bar{\kappa} \bar{\omega}\right|^{2} \\
& =-(2 \lambda-k) \int_{\Sigma}\left\langle\phi, \iota_{\nu} \bar{\omega}\right\rangle+s_{r} \int_{\Sigma}|d \phi|^{2}+s_{n-r} \int_{\Sigma}\left|i^{*} \bar{\omega}\right|^{2} .
\end{aligned}
$$

The condition $s_{q} \geq 0$ implies $s_{n-r} \geq 0$. From the above, as $\int_{\Sigma}\left\langle\phi, \iota_{\nu} \bar{\omega}\right\rangle=$ $\int_{N}|\bar{\omega}|^{2}>0$, this shows that $2 \lambda \geq k$, which proves (2.2) in the case where $s_{n-r}=0$. So in the following we can assume $s_{n-r}>0$. 
As $\left|i^{*} \bar{*} \bar{\omega}\right|^{2}=\left|\iota_{\nu} \bar{\omega}\right|^{2}$ and $\int_{\Sigma}|d \phi|^{2}=\int_{\Sigma}\langle\phi, \delta d \phi\rangle=\lambda \int_{\Sigma}|\phi|^{2}$, the inequality (2.3) becomes

$$
\begin{aligned}
0 & \geq \int_{\Sigma}-(2 \lambda-k)\left\langle\phi, \iota_{\nu} \bar{\omega}\right\rangle+s_{r} \lambda|\phi|^{2}+s_{n-r}\left|\iota_{\nu} \bar{\omega}\right|^{2} \\
& =\int_{\Sigma} s_{n-r}\left|\iota_{\nu} \bar{\omega}-\frac{\lambda-k / 2}{s_{n-r}} \phi\right|^{2}+\left(s_{r} \lambda-\frac{(\lambda-k / 2)^{2}}{s_{n-r}}\right)|\phi|^{2} \\
& \geq \int_{\Sigma}\left(s_{r} \lambda-\frac{(\lambda-k / 2)^{2}}{s_{n-r}}\right)|\phi|^{2} .
\end{aligned}
$$

As $\phi$ is not identically zero, we conclude that

$$
\left(\lambda-\frac{k}{2}\right)^{2} \geq s_{r} s_{n-r} \lambda=2 c \lambda
$$

where $2 c=s_{r} s_{n-r}$. This implies either

$$
\lambda-\frac{k}{2} \leq c-\sqrt{c^{2}+c k} \text { or } \lambda-\frac{k}{2} \geq c+\sqrt{c^{2}+c k} .
$$

In view of (2.3), we conclude that the second case holds. i.e.

$$
2 \lambda \geq k+s_{r} s_{n-r}+\sqrt{\left(s_{r} s_{n-r}\right)^{2}+2 s_{r} s_{n-r} k} .
$$

Suppose the equality holds, then from (2.3), $\bar{\nabla} \bar{\omega}=0$. As $\bar{\omega}$ is parallel, $|\bar{\omega}|^{2}$ is constant, and as $i^{*} \bar{\omega}=\omega$, this constant is nonzero, which we can assume to be 1 . The curvature term $W^{r}$ is given by (see e.g. [14] p.218 Theorem 50):

$$
W^{r}(\bar{\omega})=\frac{1}{2} \sum_{i, j=1}^{n} \theta^{i} \cdot \theta^{j} \cdot \bar{R}\left(e_{i}, e_{j}\right) \bar{\omega}
$$

where $\left\{e_{j}\right\}_{j=1}^{n}$ is a local orthonormal frame on $N,\left\{\theta^{j}\right\}_{j=1}^{n}$ is its dual frame and $\bar{R}$ is the curvature operator on $(N, g)$. Here $\theta^{i} \cdot \alpha=\theta^{i} \wedge$ $\alpha-\iota_{e_{i}} \alpha$ is the Clifford multiplication on a differential form $\alpha$. Since $0=\bar{\nabla} \bar{\omega}$, we have $\bar{\nabla}^{2} \bar{\omega}=0$ and so $\bar{R}\left(e_{i}, e_{j}\right) \bar{\omega}=0$. Therefore from (2.3)

$$
0=\frac{1}{2}\left\langle\sum_{i, j=1}^{n} \theta^{i} \cdot \theta^{j} \cdot \bar{R}\left(e_{i}, e_{j}\right) \bar{\omega}, \bar{\omega}\right\rangle=\left\langle W^{r}(\bar{\omega}), \bar{\omega}\right\rangle=k|\bar{\omega}|^{2}=k .
$$

So we now have $\lambda=s_{r} s_{n-r}>0$. Therefore from (2.4),

$$
\iota_{\nu} \bar{\omega}=\frac{\lambda}{s_{n-r}} \phi=s_{r} \phi .
$$

From this and (2.3), (2.4), we see that $S^{r} \equiv s_{r}$ and $S^{n-r} \equiv s_{n-r}$, i.e. the $r$-curvatures and the $(n-r)$-curvatures are constants. 
Now we suppose, furthermore, that $\overline{\operatorname{Ric}} \geq 0$. As $|\bar{\omega}|^{2}=1$,

$$
\begin{aligned}
\operatorname{Area}(\Sigma)=\int_{\Sigma}|\bar{\omega}|^{2}=\int_{\Sigma}\left(|\omega|^{2}+\left|\iota_{\nu} \bar{\omega}\right|^{2}\right) & =\int_{\Sigma}\left(|d \phi|^{2}+\left|\iota_{\nu} \bar{\omega}\right|^{2}\right) \\
& =\int_{\Sigma} \lambda_{1}|\phi|^{2}+\left|\iota_{\nu} \bar{\omega}\right|^{2} \\
& =\left(\frac{s_{r}+s_{n-r}}{s_{r}}\right) \int_{\Sigma}\left|\iota_{\nu} \bar{\omega}\right|^{2} .
\end{aligned}
$$

On the other hand, by Stokes theorem,

$$
\begin{aligned}
\operatorname{Vol}(N)=\int_{N}|d \bar{\phi}|^{2}=\int_{N}\langle\bar{\phi}, \bar{\delta} \bar{d} \bar{\phi}\rangle+\int_{\Sigma}\left\langle i^{*} \bar{\phi}, \iota_{\nu} \bar{d} \bar{\phi}\right\rangle & =\int_{\Sigma}\left\langle\phi, \iota_{\nu} \bar{\omega}\right\rangle \\
& =\frac{1}{s_{r}} \int_{\Sigma}\left|\iota_{\nu} \bar{\omega}\right|^{2} .
\end{aligned}
$$

From these we have

$$
\frac{\operatorname{Area}(\Sigma)}{\operatorname{Vol}(N)}=s_{r}+s_{n-r} \text {. }
$$

Recall that we have $\frac{s_{l}}{l} \leq \frac{s_{m}}{m}$ for $l \leq m$, so $s_{r}+s_{n-r} \leq \frac{r}{n-1} s_{n-1}+$ $\frac{n-r}{n-1} s_{n-1}=\frac{n}{n-1} s_{n-1}$. Thus

$$
\frac{\operatorname{Area}(\Sigma)}{\operatorname{Vol}(N)} \leq \frac{n}{n-1} s_{n-1}
$$

By [19] Theorem 1, as $\overline{\mathrm{Ric}} \geq 0$, we conclude that $(N, g)$ is isometric to a Euclidean ball.

Using $\bar{\nabla}_{X}(\bar{*} \alpha)=\bar{*}\left(\bar{\nabla}_{X} \alpha\right)$ and $\theta^{j} \cdot \bar{*} \alpha=\bar{*}\left(\theta^{j} \cdot \alpha\right)$, we have, by (2.5),

$$
\left\langle W^{r}(\bar{\omega}), \bar{\omega}\right\rangle=\left\langle W^{n-r}(\bar{*} \bar{\omega}), \bar{\omega} \bar{\omega}\right\rangle \text {. }
$$

As $W^{1}=\overline{\mathrm{Ric}}$ and $k=0$, so the condition $\overline{\mathrm{Ric}} \geq 0$ is redundant for $r=1$ or $n-1$. Finally, it is well-known that (see e.g. [10])

$$
\lambda_{1, r}^{\prime}\left(\mathbb{S}^{n-1}\right)=r(n-r) .
$$

From this it is easy to see that the equality holds on any Euclidean ball, with $k=0$.

Remark 1. When $k=0$ and $r=1$, Theorem 2.3] is Theorem 1 in [24].

To state our next result, we need to define the Steklov eigenvalues as follows. Let $\alpha \in \Omega^{r}(\Sigma), r=0, \cdots, n-1$. Then there exists a unique $r$-form $\bar{\alpha} \in \Omega^{r}(N)$ such that (see e.g. [20] Theorem 3.4.6)

$$
\left\{\begin{array}{l}
\bar{\Delta} \bar{\alpha}=0 \quad \text { on }(N, g), \\
i^{*} \bar{\alpha}=\alpha, \iota_{\nu} \bar{\alpha}=0 \quad \text { on } \Sigma .
\end{array}\right.
$$


We define the Steklov operator $T^{r}: \Omega^{r}(\Sigma) \rightarrow \Omega^{r}(\Sigma)$ by

$$
T^{r} \alpha=\iota_{\nu} \bar{d} \bar{\alpha} .
$$

By [16] Theorem 11, $T^{r}$ is an elliptic nonnegative self-adjoint pseudodifferential operator of order one. Thus the eigenvalue problem

$$
T^{r} \alpha=p \alpha
$$

has a discrete spectrum

$$
0 \leq p_{1, r}(N) \leq p_{2, r}(N) \leq \cdots .
$$

We will write $p_{k, r}$ for $p_{k, r}(N)$. Here we use the convention in [16] that $p_{1, r}$ is the smallest nonnegative eigenvalue of $T^{r}$. Thus in the classical case where $r=0$, i.e. for $f \in C^{\infty}(\Sigma), \bar{f}$ being the unique harmonic extension of $f$ to $N$ and

$$
T f=T^{0} f=\frac{\partial \bar{f}}{\partial \nu}
$$

the first nonnegative eigenvalue of $T$ is zero, corresponding to the constant functions on $\Sigma$. So in our convention, $p_{1,0}=0$ and $p_{2,0}$ is the smallest positive eigenvalue, usually called the first Steklov eigenvalue of $N$. We will simply denote $p_{2,0}$ by $p_{2}$.

We remark that the first eigenvalue of $T^{r}$ satisfies the min-max principle ([16] Theorem 11):

$$
p_{1, r}(N)=\inf \left\{\frac{\int_{N}\left(|\bar{d} \bar{\phi}|^{2}+|\bar{\delta} \bar{\phi}|^{2}\right)}{\int_{\Sigma}|\bar{\phi}|^{2}}: 0 \neq \bar{\phi} \in \Omega^{r}(N), \iota_{\nu} \bar{\phi}=0\right\} .
$$

When $r=0$, we also have the following min-max principle for the smallest nonzero Steklov eigenvalue (see for example [12] p.113):

$$
p_{2}(N)=p_{2,0}(N)=\inf \left\{\frac{\int_{N}|\bar{\nabla} \bar{\phi}|^{2}}{\left.\int_{\Sigma} \bar{\phi}\right|_{\Sigma} ^{2}}: 0 \neq \bar{\phi} \in C^{\infty}(N),\left.\int_{\Sigma} \bar{\phi}\right|_{\Sigma}=0\right\} .
$$

Remark 2. By the Hodge-deRham theorem for manifolds with boundary (20] Theorem 2.6.1), any cohomology class of the deRham cohomology space (with real coefficients) $H_{d R}^{r}(N, \bar{d})$ is uniquely represented by $\bar{\phi} \in \Omega^{r}(N)$ such that

$$
\left\{\begin{array}{l}
\bar{d} \bar{\phi}=\bar{\delta} \bar{\phi}=0 \text { on } N \\
\iota_{\nu} \bar{\phi}=0 \text { on } \Sigma
\end{array}\right.
$$


We will denote the space of all such $\bar{\phi}$ by $\mathcal{H}^{r}(N)$. So from (2.8), we see that $p_{1, r}$ is positive if and only if $\mathcal{H}^{r}(N)=0$. Therefore we are interested in $p_{1, r}$ only when $\mathcal{H}^{r}(N)=0$.

By Hodge duality, the relative deRham cohomology space (cf. [20] p.103) $H_{d R}^{r}(N, \bar{\delta})$ is isomorphic to the vector space

$$
\mathcal{H}_{R}^{r}(N)=\left\{\bar{\phi} \in \Omega^{r}(N): \bar{d} \bar{\phi}=\bar{\delta} \bar{\phi}=0 \text { on } N, i^{*} \bar{\phi}=0 \text { on } \Sigma\right\},
$$

called the space of Dirichlet harmonic fields.

Theorem 2.4. Let $\left(N^{n}, g\right)$ be a compact orientable Riemannian manifold with boundary $\Sigma$. Let $r=1, \cdots, n-1$. We assume $p_{1, r-1}$ is nontrivial if $r>1$ (corresponding to $\mathcal{H}^{r-1}(N)=0$ ). Suppose $W^{r}(N) \geq k$, the $r$-curvatures of $\Sigma$ are bounded from below by $l$ and $s_{n-r} \geq 0$. Let $\lambda=\lambda_{1, r}^{\prime}(\Sigma)=\lambda_{1, r-1}^{\prime \prime}(\Sigma)$ and let $p$ to be $p_{1, r-1}$ if $r>1$ and $p_{2}=p_{2,0}$ if $r=1$. Then

(1) We have the following upper bound for $p$ :

$$
s_{n-r} p \leq \lambda-\frac{k}{2}+\left(\left(\lambda-\frac{k}{2}\right)^{2}-s_{n-r} l \lambda\right)^{\frac{1}{2}} .
$$

(2) Assume $l \leq 0$, then we have the following lower bounds for $p$ and $\lambda$ :

$$
\begin{gathered}
s_{n-r} p \geq \lambda-\frac{k}{2}-\left(\left(\lambda-\frac{k}{2}\right)^{2}-s_{n-r} l \lambda\right)^{\frac{1}{2}} . \\
\lambda \geq \frac{s_{n-r} p^{2}+k p}{2 p-l} .
\end{gathered}
$$

(3) Assume $k \geq 0$ and $l \geq 0$. We have either

$$
\lambda \geq s_{n-r} p+\frac{k}{2}
$$

or

$$
\lambda \geq \frac{s_{n-r} p^{2}+k p}{2 p-l},
$$

provided that it is well-defined. (If $\lambda \leq s_{n-r} p+\frac{k}{2}$ and $s_{n-r}>0$, we will show that $2 p-l>0$, see Remark 3. )

(4) Assume $s_{r} \geq 0$, and $\mathcal{H}_{R}^{r}(N)=0$. Then

$$
2 \lambda \geq k+s_{r} p_{1, n-1-r}+s_{n-r} p .
$$

If $r=1$, the condition $\mathcal{H}_{R}^{1}(N)=0$ can be replaced by $s_{n-1}>0$ and $k \geq 0$. 
(5) The inequalities (2.11) and (2.12) are actually strict (if $l \leq 0$ ). Any of the equality cases in (2.10), (2.14) or (2.15) can hold only when $k=0$, with the $r$-curvatures and $(n-r)$-curvatures both being positive constants.

Suppose $(N, g)$ has non-negative Ricci curvature. Then the equality in (2.10) or (2.14) holds if and only if $r \geq \frac{n}{2}+1$ or $r=1$, and $(N, g)$ is isometric to a Euclidean ball. The condition on Ricci curvature can be removed if $r=1$. The equality case in (2.15) can hold if and only if $r=1, n \geq 4$ and $(N, g)$ is a Euclidean ball.

Proof. Let $\phi$ be a co-exact $(r-1)$-eigenform on $\Sigma$ with eigenvalue $\lambda=\lambda_{1, r-1}^{\prime \prime}=\lambda_{1, r}^{\prime}$, i.e. $\Delta \phi=-\delta d \phi=-\lambda \phi$. Then $\omega=d \phi$ is an exact $r$-eigenform on $\Sigma$ and by [20] Lemma 3.4.7, there exists an $(r-1)$-form $\bar{\phi}$ on $N$ such that

$$
\left\{\begin{array}{l}
-\bar{\Delta} \bar{\phi}=(\bar{d} \bar{\delta}+\bar{\delta} \bar{d}) \bar{\phi}=0 \quad \text { on } N \\
i^{*} \bar{\phi}=\phi, \quad i^{*} \bar{\delta} \bar{\phi}=0 \quad \text { on } \Sigma
\end{array}\right.
$$

By Stokes theorem,

$$
\int_{N}|\bar{d} \bar{\delta} \bar{\phi}|^{2}=\int_{N}\langle\bar{\delta} \bar{\phi}, \bar{\delta} \bar{d} \bar{\delta} \bar{\phi}\rangle+\int_{\Sigma}\left\langle i^{*} \bar{\delta} \bar{\phi}, \iota_{\nu} \bar{d} \overline{\delta \phi}\right\rangle=\int_{N}\langle\bar{\delta} \bar{\phi},-\bar{\delta} \bar{\delta} \bar{d} \bar{\phi}\rangle=0 .
$$

So we have $\bar{d} \bar{\delta} \bar{\phi}=\bar{\delta} \bar{d} \bar{\phi}=0$. Let $\bar{\omega}=\bar{d} \bar{\phi}$, then $\bar{\omega}$ is a harmonic field, i.e. $\bar{d} \bar{\omega}=\bar{\delta} \bar{\omega}=0$.

By applying Reilly's formula (Theorem 2.1) on $\bar{\omega}=\bar{d} \bar{\phi}$, and following exactly the same steps in the proof of Theorem 2.3 ,

$$
0 \geq \int_{N}-|\bar{\nabla} \bar{\omega}|^{2} \geq-(2 \lambda-k) \int_{\Sigma}\left\langle\phi, \iota_{\nu} \bar{\omega}\right\rangle+l \lambda \int_{\Sigma}|\phi|^{2}+s_{n-r} \int_{\Sigma}\left|\iota_{\nu} \bar{\omega}\right|^{2} .
$$

We now prove (11) and (2) together. Let us first assume $l \geq 0$. As $\omega \neq 0, \int_{\Sigma}\left\langle\phi, \iota_{\nu} \bar{\omega}\right\rangle=\int_{N}|\bar{\omega}|^{2}>0$, thus by (2.16), we have

$$
2 \lambda-k \geq 0 \text {. }
$$

The inequality (2.10) (and also (2.11)) is trivial if $s_{n-r}=0$, so we assume $s_{n-r}>0$. Let $k=2 a, U=\left(\int_{\Sigma}\left|\iota_{\nu} \bar{\omega}\right|^{2}\right)^{\frac{1}{2}}$ and $Z=\left(\int_{\Sigma}|\phi|^{2}\right)^{\frac{1}{2}}>0$. So by Cauchy Schwarz inequality,

$$
s_{n-r} U^{2}+l \lambda Z^{2} \leq 2(\lambda-a) \int_{\Sigma}\left\langle\phi, \iota_{\nu} \bar{\omega}\right\rangle \leq 2(\lambda-a) U Z .
$$


By completing the square,

$$
s_{n-r} \frac{U}{Z} \leq \lambda-a+\left((\lambda-a)^{2}-s_{n-r} l \lambda_{1}\right)^{\frac{1}{2}} .
$$

Let us for the time being assume $r>1$. We claim that

$$
p_{1, r-1} \leq \frac{\int_{\Sigma}\left\langle\phi, \iota_{\nu} \bar{\omega}\right\rangle}{\int_{\Sigma}|\phi|^{2}}
$$

By the Friedrichs decomposition for harmonic fields, as $\bar{\omega}$ is exact, there is a unique co-exact $(r-1)$-form $\widetilde{\phi}$ on $N$ such that (see [20] Theorem 2.4.8 and its proof):

$$
\bar{d} \widetilde{\phi}=\bar{\omega} \quad \text { on } N, \quad \iota_{\nu} \widetilde{\phi}=0 \quad \text { on } \Sigma .
$$

Let $\phi^{\prime}=i^{*} \widetilde{\phi}$, then as $\bar{\delta} \widetilde{\phi}=0$,

$$
\int_{\Sigma}\left\langle\phi^{\prime}, \iota_{\nu} \bar{\omega}\right\rangle=\int_{N}\langle\bar{d} \widetilde{\phi}, \bar{\omega}\rangle-\langle\bar{\phi}, \bar{\delta} \bar{\omega}\rangle=\int_{N}|\bar{d} \widetilde{\phi}|^{2}=\int_{N}|\bar{d} \widetilde{\phi}|^{2}+|\bar{\delta} \widetilde{\phi}|^{2} .
$$

Thus by (2.8),

$$
p_{1, r-1} \leq \frac{\int_{\Sigma}\left\langle\phi^{\prime}, \iota_{\nu} \bar{\omega}\right\rangle}{\int_{\Sigma}\left|\phi^{\prime}\right|^{2}}
$$

On the other hand, we have

$$
\int_{\Sigma}\left\langle\phi^{\prime}, \iota_{\nu} \bar{\omega}\right\rangle=\int_{N}|\bar{d} \widetilde{\phi}|^{2}=\int_{N}|\bar{d} \bar{\phi}|^{2}=\int_{\Sigma}\left\langle\phi, \iota_{\nu} \bar{\omega}\right\rangle .
$$

As $\bar{d} \widetilde{\phi}=\bar{d} \bar{\phi}$, we also have $d \phi^{\prime}=d \phi$, so

$$
\lambda \int_{\Sigma}|\phi|^{2}=\int_{\Sigma}|d \phi|^{2}=\int_{\Sigma}\left\langle d \phi^{\prime}, d \phi\right\rangle=\int_{\Sigma}\left\langle\phi^{\prime}, \delta d \phi\right\rangle=\lambda \int_{\Sigma}\left\langle\phi^{\prime}, \phi\right\rangle .
$$

We conclude that $\phi^{\prime}-\phi \perp \phi$ and thus $\int_{\Sigma}\left|\phi^{\prime}\right|^{2} \geq \int_{\Sigma}|\phi|^{2}$. Combining this with (2.22), (2.21), we can get (2.20). By Cauchy Schwarz inequality,

$$
p_{1, r-1} \leq \frac{\int_{\Sigma}\left\langle\phi, \iota_{\nu} \bar{\omega}\right\rangle}{\int_{\Sigma}|\phi|^{2}} \leq \frac{\int_{\Sigma}\left|\iota_{\nu} \bar{\omega}\right|^{2}}{\int_{\Sigma}\left\langle\phi, \iota_{\nu} \bar{\omega}\right\rangle}
$$

which implies

$$
p_{1, r-1}^{2} \leq \frac{U^{2}}{Z^{2}}
$$

Putting this into (2.19), we obtain (2.10)

$$
s_{n-r} p_{1, r-1} \leq \lambda-a+\left((\lambda-a)^{2}-s_{n-r} l \lambda\right)^{\frac{1}{2}} .
$$

We now claim that this is also true for $l \leq 0$. Actually, in this case, by (2.16) and (2.23),

$$
2 \lambda-k \geq s_{n-r} \frac{\int_{\Sigma}\left|\iota_{\nu} \bar{\omega}\right|^{2}}{\int_{\Sigma}\left\langle\phi, \iota_{\nu} \bar{\omega}\right\rangle}+l \lambda \frac{\int_{\Sigma}|\phi|^{2}}{\int_{\Sigma}\left\langle\phi, \iota_{\nu} \bar{\omega}\right\rangle} \geq s_{n-r} p_{1, r-1}+\frac{l \lambda}{p_{1, r-1}}
$$


Rearranging, we have

$$
s_{n-r} p_{1, r-1}^{2}+k p_{1, r-1} \leq\left(2 p_{1, r-1}-l\right) \lambda
$$

which implies (2.10) and (2.11) (regardless of whether $s_{n-r}=0$ ). Also, (2.12) follows immediately from (2.25).

We have completed the proofs of (1) and (2) except for the case where $r=1$. For $r=1$, the proofs proceed in the same way except we have to replace (2.23) by

$$
p_{2}=p_{2,0}(N) \leq \frac{\int_{\Sigma}\left\langle i^{*} \bar{\phi}, \iota_{\nu} \bar{\omega}\right\rangle}{\int_{\Sigma}|\phi|^{2}} \leq \frac{\int_{\Sigma}\left|\iota_{\nu} \bar{\omega}\right|^{2}}{\int_{\Sigma}\left\langle i^{*} \bar{\phi}, \iota_{\nu} \bar{\omega}\right\rangle} .
$$

This is true due to the min-max principle for $p_{2}$ (Equation (2.9)), together with the fact that $\int_{\Sigma}\left\langle i^{*} \bar{\phi}, \iota_{\nu} \bar{d} \bar{\phi}\right\rangle=\int_{N}\left(|\bar{\nabla} \bar{\phi}|^{2}+\bar{\phi} \bar{\Delta} \bar{\phi}\right)=\int_{N}|\bar{\nabla} \bar{\phi}|^{2}$ and $\int_{\Sigma} \phi=-\frac{1}{\lambda} \int_{\Sigma} \Delta \phi=0$.

We now prove (3). If $s_{n-r}=0$, then (2.13) becomes $\lambda \geq \frac{k}{2}$ which is true in view of (2.17). We can now assume $s_{n-r}>0$. Suppose $\lambda-\frac{k}{2} \leq s_{n-r} p$, then by (2.10), we have

$$
0 \leq s_{n-r} p-\left(\lambda-\frac{k}{2}\right) \leq\left(\left(\lambda-\frac{k}{2}\right)^{2}-s_{n-r} l \lambda\right)^{\frac{1}{2}} .
$$

Squaring this inequality gives $s_{n-r} p^{2}+k p \leq(2 p-l) \lambda$. From this we see that $p>\frac{l}{2}$ and (2.13) follows.

For (4), we can put $l=s_{r}$ in (2.3) and using (2.23) or (2.26) to obtain

$$
2 \lambda-k \geq s_{n-r} p+s_{r} \frac{\int_{\Sigma}\left|i^{*} \bar{\omega}\right|^{2}}{\int_{\Sigma}\left\langle\iota_{\nu} \bar{\omega}, \phi\right\rangle}=s_{n-r} p+s_{r} \frac{\int_{\Sigma}\left|i^{*} \bar{\omega}\right|^{2}}{\int_{N}|\bar{\omega}|^{2}} .
$$

As $\bar{\omega}$ is co-closed and $\mathcal{H}_{R}^{r}(N) \cong H_{d R}^{r}(N, \bar{\delta})=0$, it is also co-exact. So by [16] Proposition 14, $\frac{\int_{\Sigma}\left|i^{*} \bar{\omega}\right|^{2}}{\int_{N}|\bar{\omega}|^{2}} \geq p_{1, n-1-r}$, and (2.15) follows. If $r=1$, $k \geq 0$ and $s_{n-1}>0$, then by [20] (Theorem 2.6.4, Corollary 2.6.2 and Theorem 2.6.1), $\mathcal{H}_{R}^{1}(N)=0$, thus this later condition can be dropped.

We now prove (5). Suppose the equality sign in any of the inequalities (2.10), (2.11), (2.12), (2.14) and (2.15) holds, then by $(2.3), \bar{\nabla} \bar{\omega}=0$. We can then argue as in the proof of Theorem 2.3 that $k=0$.

If any inequality sign of the inequalities (2.10), (2.11), (2.12) or (2.14) becomes an equality sign, then one of the inequalities in (2.10) or (2.11) is an equality. Assume one of these holds. The inequalities (2.23) (or $(2.26))$ and (2.3) then become equations. So we have the $r$-curvatures 
are constantly equal to $s_{r}=l, \iota_{\nu} \bar{\omega}=p \phi$ and the $(n-r)$-curvatures are equal to the constant $s_{n-r}$. In particular, $S^{n-r} \equiv s_{n-r}$.

We now show that $\lambda=s_{n-r} s_{r}$. To do this we make use of the following formulas:

$$
\begin{cases}\delta i^{*} \bar{\alpha}=i^{*} \bar{\delta} \bar{\alpha}+\iota_{\nu} \bar{\nabla}_{\nu} \bar{\alpha}-S^{r-1}\left(\iota_{\nu} \bar{\alpha}\right)+H \iota_{\nu} \bar{\alpha} & \text { for } \bar{\alpha} \in \Omega^{r}(N), \\ * S^{r}(\alpha)+S^{n-1-r}(* \alpha)=H * \alpha & \text { for } \alpha \in \Omega^{r}(\Sigma), \\ * * \alpha=(-1)^{(n-1-r) r} \alpha & \text { for } \alpha \in \Omega^{r}(\Sigma), \\ \bar{\delta} \bar{\alpha}=-\sum_{j=1}^{n} \iota_{e_{j}} \bar{\nabla}_{e_{j}} \bar{\alpha} & \text { for } \bar{\alpha} \in \Omega^{r}(N) .\end{cases}
$$

Here $*: \Omega^{r}(\Sigma) \rightarrow \Omega^{n-1-r}(\Sigma)$ is the Hodge star operator on $\Sigma$ and $\left\{e_{j}\right\}_{j=1}^{n}$ is a local orthonormal frame on $N$. The last two formulas are standard and are included here just for convenience (e.g. [20]). For the first two formulas, see [15] Section 2 and 6. Using (2.28), we compute

$$
\begin{aligned}
\delta d i^{*} \bar{\phi} & =\delta i^{*} \bar{d} \bar{\phi}=\delta i^{*} \bar{\omega} \\
& =i^{*} \bar{\delta} \bar{\omega}+\iota_{\nu} \bar{\nabla}_{\nu} \bar{\omega}-S^{r-1}\left(\iota_{\nu} \bar{\omega}\right)+H \iota_{\nu} \bar{\omega} \\
& =i^{*}\left(\sum_{j=1}^{n} \iota_{e_{j}} \bar{\nabla}_{e_{j}} \bar{\omega}\right)-S^{r-1}\left(\iota_{\nu} \bar{\omega}\right)+\left((-1)^{n(r-1)} * S^{n-r}\left(* \iota_{\nu} \bar{\omega}\right)+S^{r-1}\left(\iota_{\nu} \bar{\omega}\right)\right) \\
& =(-1)^{n(r-1)} * S^{n-r}\left(* \iota_{\nu} \bar{\omega}\right) \\
& =(-1)^{n(r-1)} s_{n-r} * * \iota_{\nu} \bar{\omega} \\
& =s_{n-r} \iota_{\nu} \bar{\omega} .
\end{aligned}
$$

This implies

$$
-\lambda \phi+s_{n-r} p \phi=-(d \delta+\delta d) i^{*} \bar{\phi}+s_{n-r} \iota_{\nu} \bar{\omega}=-\delta d i^{*} \bar{\phi}+s_{n-r} \iota_{\nu} \bar{\omega}=0
$$

As $s_{n-r} p=\lambda \pm\left(\lambda^{2}-s_{n-r} s_{r} \lambda\right)^{\frac{1}{2}}$, we conclude that $-\lambda+\lambda \pm\left(\lambda^{2}-\right.$ $\left.s_{n-r} s_{r} \lambda\right)^{\frac{1}{2}}=0$, or

$$
\lambda=s_{n-r} s_{r}=s_{n-r} p>0 .
$$

This shows that $s_{r}=l>0$ which contradicts the assumption of (2), thus the inequalities (2.11) and (2.12) must be strict.

We can now proceed in exactly the same way as the proof of Theorem 2.3 to show that $N$ must be a Euclidean ball if $\overline{\mathrm{Ric}} \geq 0$, which we can w.l.o.g. assume to be the standard unit ball $\mathbb{B}^{n}$. But then by [17] Corollary 4, 


$$
p_{1, r-1}\left(\mathbb{B}^{n}\right)=\left\{\begin{array}{l}
r \quad \text { if } r \geq \frac{n}{2}+1, \\
\frac{n+2}{n}(r-1) \quad \text { if } 2 \leq r \leq \frac{n}{2}+1 .
\end{array}\right.
$$

As $s_{m}\left(\mathbb{S}^{n-1}\right)=m$ and by (2.7), we conclude that if $r>1$, the equality in (2.10) or (2.14) holds if and only if $r \geq \frac{n}{2}+1$. For $r=1$, it is well-known that $p_{2,0}\left(\mathbb{B}^{n}\right)=1$, from this we can also conclude that the equality in (2.10) or (2.14) holds if and only if $N$ is a Euclidean ball.

Suppose the equality in (2.15) holds, then by (2.23) or (2.26),$\iota_{\nu} \bar{\omega}=$ $p \phi$ and by the same reason as above, the $r$-curvatures are constantly equal to $s_{r}$, the $(n-r)$-curvatures are constantly $s_{n-r}$, and $\lambda=s_{n-r} p$. In particular, $s_{r} \neq 0$ in view of (2.15), so from (2.27), we have

$$
p_{1, n-1-r}=\frac{\int_{\Sigma}\left|i^{*} \bar{\omega}\right|^{2}}{\int_{N}|\bar{\omega}|^{2}}=\frac{\lambda \int_{\Sigma}|\phi|^{2}}{\int_{\Sigma}\left\langle\iota_{\nu} \bar{\omega}, \phi\right\rangle}=\frac{\lambda}{p} .
$$

In view of (2.15), we deduce that $p=s_{r}$. We can then proceed as before to conclude that if $\overline{\mathrm{Ric}} \geq 0$, then $(N, g)$ is a Euclidean ball. But then by (2.7) and (2.29), the equality cannot be attained on a Euclidean ball if $r>1$. If $r=1$, then from (2.29) we see that the equality is attained if and only if $n \geq 4$, on a Euclidean ball.

Remark 3. (1) Escobar ([6] Theorem 8) showed that if $k \geq 0$, then $p_{2,0}>\frac{s_{1}}{2}$, so (2.14) is well-defined. Also, (2.15) is a generalization [4, Theorem 9] and [16, Theorem 8, Theorem 9].

(2) Theorem 2.4 (11) is an extension of [22] Theorem 1.1, in which they provided an upper bound for $p_{2}$, which corresponds to our result when $k=0$ and $r=1$.

(3) We suspect that (2.14) holds whenever $k \geq 0$ and in this case we have $2 p>s_{r} \geq l$, but we are unable to show it for the time being.

\section{SOME APPLICATIONS AND SPECIAL CASES}

In [25], Yang and Yau proved that for a compact Riemann surface $\Sigma$ of genus $g$, for any metric on $\Sigma, \lambda_{1}(\Sigma) \operatorname{Area}(\Sigma) \leq 8 \pi(1+g)$. Combining this result with Theorem 2.3 and [2.4, we have several corollaries. Let us only state the following:

Corollary 3.1. If $N=\mathbb{S}^{3}$, then under the assumptions of Theorem 2.3, we have $\left(2+s_{n-1} s_{1}+\sqrt{\left(s_{n-1} s_{1}\right)^{2}+4 s_{n-1} s_{1}}\right) \operatorname{Area}(\Sigma)<16 \pi(g+1)$. 
Remark 4. Although the estimate of Theorem 2.3 is not sharp when $k \neq 0, r=1$, by examining the case where $\Sigma$ is a geodesic circle of radius $\rho$ in a hemisphere $\left(\lambda_{1}=\lambda_{1,0}^{\prime \prime}(\Sigma)=1 / \sin ^{2}(\rho)\right)$, it is found that the error is within $1 / 2$. Indeed, in this case, $k=1, s_{1}=s_{n-1}=\cot \rho$, we have $\lambda_{1}-\frac{1}{2}\left(k+s_{1}^{2}+\sqrt{s_{1}^{4}+2 s_{1}^{2} k}\right)=\frac{1}{2} \csc ^{2} \rho-\frac{1}{2} \sqrt{\csc ^{4} \rho-1} \leq \frac{1}{2}$. The error tends to zero as $\rho \rightarrow 0$.

On the other hand, by [6] Example 5, the first nonzero Steklov eigenvalue of the geodesic ball of radius $\rho$ in $\mathbb{S}^{2}$ is computed to be $\cot \rho+\tan \frac{\rho}{2}$. By direct computations, it is found that the error in Theorem 2.4 (2) is $\lambda_{1}-\frac{s_{1} p_{2}^{2}+k p_{2}}{2 p_{2}-s_{1}}=\frac{\tan ^{2}(\rho / 2)}{2-\cos \rho}$ which is (very) slightly better than that of Theorem 2.3.

The following result is another immediate consequence of Theorem 2.3, which can be regarded as the analogue of Theorem 2 of Hang-Wang [11] (see also [24] Corollary 1).

Corollary 3.2. Let $\left(N^{n}, g\right)$ be a compact orientable Riemannian manifold with boundary $\Sigma$. Suppose the Ricci curvature of $N$ is nonnegative, $s_{r}(\Sigma) s_{n-r}(\Sigma) \geq r(n-r)=\lambda_{1, r-1}^{\prime \prime}\left(\mathbb{S}^{n-1}\right) \geq \lambda_{1, r-1}^{\prime \prime}(\Sigma)$ for some $r=1, \cdots, n-1$, and $W^{r}$ is nonnegative, then $(N, g)$ is isometric to the unit ball in $\mathbb{R}^{n}$.

Theorem 2.3 gives a quick proof of the following result, which is the $K \geq 0$ analogue of Theorem 3.1 ;

Corollary 3.3. Suppose $\left(N^{2}, g\right)$ be a compact surface with (not necessarily connected) boundary $\gamma$ with the Gaussian curvature $K \geq 0$. If the geodesic curvature $k_{g}$ of $\gamma$ satisfies $k_{g} \geq l>0$, then its length $L(\gamma) \leq \frac{2 \pi}{l}$. The equality holds if and only if $(N, g)$ is isometric to the Euclidean disk of radius $1 / l$.

Proof. By Gauss-Bonnet theorem, $2 \pi \chi(N)=\int_{N} K+\int_{\gamma} k_{g}>0$, thus $\gamma$ has only one component. By Theorem $2.3, \lambda_{1}(\gamma) \geq l^{2}$. The equality holds if and only if $N$ is a Euclidean disk of radius $1 / l$. As $\lambda_{1}(\gamma)=$ $\left(\frac{2 \pi}{L(\gamma)}\right)^{2}$, the result follows.

In [3], Choi and Wang proved that if $\left(N^{n}, g\right)$ is a compact orientable manifold whose Ricci curvature is bounded from below by $k>0$ and $\Sigma$ is an embedded orientable minimal hypersurface in $N$, then $\lambda_{1}(\Sigma) \geq \frac{k}{2}$. Since their proof are essentially the same as that of Theorem 2.3, their result can be improved slightly to $\lambda_{1}(\Sigma)>\frac{k}{2}$. This is related to Yau's conjecture [26]. It is easy to see that the coordinate functions are eigenfunctions of a minimal hypersurface of $\mathbb{S}^{n}$ (whose Ricci curvature 
is $n-1$ ) with eigenvalue $n-1$. Yau conjectured that the first eigenvalue is actually $n-1$. Escobar also have a similar conjecture in [4]. We also notice that Barros and Bessa [1] proved an improvement on the ChoiWang estimate.

In the two-dimensional case, an embedded minimal submanifold is reduced to a simple closed geodesic, the result of Choi-Wang can be improved to $\lambda_{1} \geq k$, by a result of Toponogov [21] on the length of a closed geodesic. More generally, we have the following result which is an extension of the result in [11, which may have some independent interest:

Theorem 3.1. Let $\left(N^{2}, g\right)$ be a closed surface with Gaussian curvature $K \geq 1$. Let $\gamma$ be a simple closed curve in $N$ which separates $N$ into $N_{1}, N_{2}$. Suppose its geodesic curvature w.r.t. the outward normal of $N_{1}$ satisfies $k_{g} \geq l \geq 0$. Then its length $L(\gamma) \leq \frac{2 \pi}{\sqrt{1+l^{2}}}$, which is equivalent to $\lambda_{1}(\gamma) \geq 1+l^{2}$ (as $\left.\lambda_{1}(\gamma)=\left(\frac{2 \pi}{L(\gamma)}\right)^{2}\right)$, and also Area $\left(N_{2}\right) \leq$ Area $\left(B_{r}\right)$, where $B_{r}$ is the disk of radius $r=\cot ^{-1}(-l)$ in the standard sphere $\mathbb{S}^{2}$. If $L(\gamma)=\frac{2 \pi}{\sqrt{1+l^{2}}}$ then $N_{1}$ is isometric to $B_{\pi-r}$. If, moreover, Area $\left(N_{2}\right)=\operatorname{Area}\left(B_{r}\right)$, then $(N, g)$ is $\mathbb{S}^{2}$. The condition for the area can be dropped if $l=0$.

Proof. By [11] Theorem 3, we have $L(\gamma) \leq \frac{2 \pi}{\sqrt{1+l^{2}}}$. The equality holds if and only if $\left(N_{1}, g\right)$ is isometric to the disk $B_{r^{\prime}} \subset \mathbb{S}^{2}, r^{\prime}=\cot ^{-1}(l)$. Therefore if $L(\gamma)=2 \pi,\left(N_{1}, g\right)$ is isometric to the standard hemisphere. But then $k_{g}=0$, thus we can apply the same argument to $N_{2}$ to deduce that $(N, g)$ is $\mathbb{S}^{2}$. In general, if $L(\gamma)=\frac{2 \pi}{\sqrt{1+l^{2}}}$, then by Gauss-Bonnet theorem, as $N$ is a topological sphere,

$$
\begin{aligned}
\operatorname{Area}\left(B_{r}\right)+\operatorname{Area}\left(B_{r^{\prime}}\right)=4 \pi=\int_{N_{2}} K+\int_{N_{1}} K & =\int_{N_{2}} K+\operatorname{Area}\left(B_{r^{\prime}}\right) \\
& \geq \operatorname{Area}\left(N_{2}\right)+\operatorname{Area}\left(B_{r^{\prime}}\right) .
\end{aligned}
$$

So if Area $\left(N_{2}\right)=\operatorname{Area}\left(B_{r}\right)$, then $K=1$ on $N$ and so $(N, g)$ is $\mathbb{S}^{2}$.

\section{REFERENCES}

1. Barros, A. and Bessa, G. P. Estimates of the first eigenvalue of minimal hypersurfaces of $\mathbb{S}^{n}$, arXiv preprint math/0410493, 2004.

2. Duff, G.F.D. and Spencer, D.C. Harmonic tensors on Riemannian manifolds with boundary, The Annals of Mathematics 56 (1), 128-156, 1952.

3. Choi, H.I. and Wang, A.N. A first eigenvalue estimate for minimal hypersurfaces, J. Differential Geometry 18 (3), 559-562, 1983.

4. Escobar, J.F. An isoperimetric inequality and the first Steklov eigenvalue, Journal of Functional Analysis 165 (1), 101-116, 1999. 
5. Fan, X-Q., Tam, L-F., and Yu C. Steklov eigenvalues on annulus, arXiv preprint arXiv:1310.7686, 2013.

6. Escobar, J.F. The geometry of the first non-zero Stekloff eigenvalue, Journal of Functional Analysis 150 (2), 544-556, 1997.

7. Fraser, A. and Schoen, R. The first steklov eigenvalue, conformal geometry, and minimal surfaces, Advances in Mathematics, 226(5): 4011-4030, 2011.

8. Fraser, A. and Schoen, R. Minimal surfaces and eigenvalue problems, Geometric Analysis, Mathematical Relativity, and Nonlinear Partial Differential Equations, 599:105-121, 2012.

9. Fraser, A. and Schoen, R. Sharp eigenvalue bounds and minimal surfaces in the ball, Inventiones Mathematicae, pages 1-68, 2012.

10. Gallot, S. and Meyer, D. Opérateur de courbure et laplacien des formes différentielles dune variété Riemannienne, J. Math. Pures et Appl 54, 259-284, 1975.

11. Hang, F. and Wang, X. A Rigidity Theorem for the Hemisphere, arXiv preprint arXiv:0711.4595.

12. Henrot, A. Extremum problems for eigenvalues of elliptic operators, Birkhauser (2006).

13. Ilias, S. and Makhoul, O. A Reilly inequality for the first Steklov eigenvalue, Differential Geometry and its Applications 29 (5), 699-708, 2011.

14. Petersen, P. Riemannian geometry, Graduate Texts in Mathematics, (1998).

15. Raulot, S. and Savo, A. A Reilly formula and eigenvalue estimates for differential forms, Journal of Geometric Analysis 21 (3), 620-640, 2011.

16. Raulot, S. and Savo, A. On the first eigenvalue of the Dirichlet-to-Neumann operator on forms, Journal of Functional Analysis 262 (3), 889-914, 2012.

17. Raulot, S. and Savo, A. On the spectrum of the Dirichlet-to-Neumann operator acting on forms of a Euclidean domain, Journal of Geometry and Physics 77: 1-12, 2014.

18. Reilly, R.C. Applications of the Hessian operator in a Riemannian manifold, Indiana Univ. Math. J. 26, 459-472, 1977.

19. Ros, A. Compact hypersurfaces with constant higher order mean curvatures, Revista Matemática Iberoamericana 3 (3), 447-453, 1987.

20. Schwarz, G. Hodge decomposition: a method for solving boundary value problems, Springer Lecture Notes in Mathematics, (1995).

21. Toponogov, V. Evaluation of the length of a closed geodesic on a convex surface. (Russian) Dokl. Akad. Nauk SSSR 124, 282-284, 1959.

22. Wang, Q. and Xia, C. Sharp bounds for the first non-zero Stekloff eigenvalues, Journal of Functional Analysis 257 (8), 2635-2644, 2009.

23. Warner, F.W. Foundations of differentiable manifolds and Lie groups, Springer Verlag, (1971).

24. Xia, C. Rigidity of compact manifolds with boundary and nonnegative Ricci curvature, Proceedings of the American Mathematical Society 125, 1801-1806, 1997.

25. Yang, P. and Yau, S. T. Eigenvalues of the Laplacian of compact Riemann surfaces and minimal submanifolds Ann. Scuola Norm. Sup. Pisa Cl. Sci.(4) 7 (1), 55-63, 1980.

26. Yau, S. T. Seminar on differential geometry, Ann. of Math. Studies (102), 669706, 1982. 
Department of Mathematics, National Cheng Kung University, Tainan CITY 70101, TAIWAN

E-mail address: kwong@mail.ncku.edu.tw 